\title{
Comparison of two different dosages of celecoxib with diclofenac for the treatment of active ankylosing spondylitis: results of a 12-week randomised, double-blind, controlled study
}

\author{
J Sieper, ${ }^{1}$ T Klopsch, ${ }^{2}$ M Richter, ${ }^{3}$ A Kapelle, ${ }^{4}$ M Rudwaleit, ${ }^{1}$ S Schwank, ${ }^{5}$ E Regourd ${ }^{5}$ \\ M May ${ }^{5}$
}

${ }^{1}$ Benjamin Franklin University Berlin, Germany; ${ }^{2}$ Rheumatology Practice, Neubrandenburg, Germany; ${ }^{3}$ Rheumatology Practice, Rostock, Germany; ${ }^{4}$ Rheumatology Practice Hoyerswerda, Germany; ${ }^{5}$ Pfizer Pharma GmbH Karlsruhe, Germany

Correspondence to:

Professor Dr J Sieper, Benjamin Franklin University Berlin, Medizinische Klinik und Poliklinik, Abteilung Rheumatologie, Hindenburgdamm 30, D-12200 Berlin, Germany; joachim. sieper@charite.de

Accepted 21 June 2007 Published Online First 6 July 2007

\section{ABSTRACT \\ Objectives: To demonstrate the non-inferiority of celecoxib compared with diclofenac in subjects with ankylosing spondylitis (AS).}

Methods: The basis of the present work was a 12-week randomised, double-blind, controlled study in active AS subjects with three treatment arms: celecoxib $200 \mathrm{mg}$ once a day, celecoxib $200 \mathrm{mg}$ twice a day, and diclofenac SR $75 \mathrm{mg}$ twice a day. The primary efficacy endpoint was the change from baseline in global pain intensity on a visual analogue scale (VAS) at week 12. Secondary endpoints covered changes in disease activity, functional and mobility capacities, and adverse events.

Results: A total of 458 subjects were randomly assigned to either celecoxib $200 \mathrm{mg}$ once a day $(n=153)$, celecoxib $200 \mathrm{mg}$ twice a day $(\mathrm{n}=150)$, or diclofenac $(n=155)$. Least square (LS) mean changes from baseline at week 12 on a pain VAS were clinically relevant in all treatment groups (celecoxib $200 \mathrm{mg}$ once a day: $-29.1 \mathrm{~mm}$; celecoxib $200 \mathrm{mg}$ twice a day: $-31.7 \mathrm{~mm}$; diclofenac: $-32.7 \mathrm{~mm}$ ) and non-inferior when compared to diclofenac. Ankylosing Spondylitis Assessment Study group 20\% (ASAS 20) response and mean improvement in Bath Ankylosing Spondylitis Disease Activity Index (BASDAI) scores at week 12 were numerically better on celecoxib $200 \mathrm{mg}$ twice a day (59.7\% and -1.32 points) and on diclofenac (60.2\% and -1.48 points) than on celecoxib $200 \mathrm{mg}$ once a day $(46.0 \%$ and -0.99 points). The incidence of gastrointestinal adverse events was significantly higher on diclofenac $(28.4 \%)$ than on celecoxib $200 \mathrm{mg}$ once a day (15.0\%) or $200 \mathrm{mg}$ twice a day $(16.7 \%)$.

Conclusions: The efficacy of celecoxib $200 \mathrm{mg}$ once a day and $200 \mathrm{mg}$ twice a day was comparable to that of diclofenac $75 \mathrm{mg}$ twice a day with respect to pain reduction. Celecoxib $200 \mathrm{mg}$ twice a day and diclofenac reduced some parameters associated with inflammation more effectively than celecoxib $200 \mathrm{mg}$ once a day. Treatment was well tolerated, with celecoxib (either dose) exhibiting less frequent gastrointestinal adverse events than diclofenac.

Non-steroidal anti-inflammatory drugs (NSAIDs) effectively improve ankylosing spondylitis (AS) symptoms. ${ }^{1} \quad$ Recent Ankylosing Spondylitis Assessment Study (ASAS) working group/ European League Against Rheumatism (EULAR) recommendations for the management of AS state that NSAIDs and the new group of tumour necrosis factor (TNF) $\alpha$ blockers are the only effective drug treatment for active $\mathrm{AS} .^{2}$ In contrast to the case in rheumatoid arthritis (RA), disease modifying antirheumatic drugs (DMARDs) such as methotrexate do not play a role in the treatment of predominant axial manifestations of $\mathrm{AS}^{2}$ Furthermore, the international ASAS recommendations for the treatment of AS call for the failure of at least two NSAIDs before patients are considered candidates for therapy with TNFa blockers. ${ }^{3}$ Thus, NSAIDs are the cornerstone in the treatment of AS, and should be considered first-line therapy

The development of NSAIDs with highly selective COX-2 inhibition such as celecoxib has raised the hope of having a drug class with an efficacy similar to that of conventional NSAIDs, but with a more favourable gastrointestinal (GI) safety profile. Therefore, several studies have addressed the GI tolerability of celecoxib compared with that of conventional NSAIDs. The Celecoxib Long-term Arthritis Safety Study (CLASS) showed that treatment with celecoxib was associated with a lower incidence of symptomatic ulcers and ulcer complications combined than conventional NSAIDs but did not demonstrate conclusively a significant reduction in upper ulcer complications alone. ${ }^{4}$ Pooled analyses in subjects with RA or osteoarthritis indicated that the upper GI tolerability (based on a composite endpoint including severe abdominal pain, dyspepsia, and nausea) was superior to that of naproxen and that there was a lower incidence of upper GI ulcer complications (ie, bleeding, perforation, and gastric outlet obstruction) under celecoxib than with a conventional NSAIDs such as naproxen, diclofenac, or ibuprofen. ${ }^{56}$

Concern has arisen in the last few years regarding a potential increase in thromboembolic events in patients taking COX-2-selective inhibitors, as compared with those taking nonspecific NSAIDs. The data with celecoxib are controversial. ${ }^{7}$ However, in randomised controlled clinical OA or RA trials no significant differences in cardiovascular events between patients treated with up to $400 \mathrm{mg}$ celecoxib/day and conventional NSAIDs could be observed. ${ }^{4}$ Two recent studies on celecoxib for the prevention of colorectal adenomas described a slightly increased cardiovascular risk for celecoxib at higher doses of 400 and $800 \mathrm{mg} /$ day in comparison with placebo. ${ }^{9}{ }^{10}$ However, this risk was not increased compared to 
non-selective NSAIDs such as diclofenac. ${ }^{11}$ As with other COX2-specific inhibitors, there are still no results from studies available that were designed or powered to evaluate especially cardiovascular adverse events.

Celecoxib was superior to placebo and was as efficacious as ketoprofen over 6 weeks or naproxen over 12 weeks for the treatment of AS. ${ }^{12} 13$ Therefore, we investigated the efficacy and safety of celecoxib at daily doses of $200 \mathrm{mg}$ once daily or $200 \mathrm{mg}$ twice daily compared with diclofenac at a high dose of $75 \mathrm{mg}$ twice a day over 12 weeks in order to further establish the therapeutic utility of celecoxib in the treatment of AS.

\section{PATIENTS AND METHODS}

\section{Study design and subject population}

We conducted a multicentre, randomised, controlled, doubleblind study over 12 weeks at 47 investigational centres (rheumatologists in outpatient units or in private practices) across Germany to compare two different doses of celecoxib versus high dose diclofenac in subjects with AS. Approval from the local Ethics Committees was obtained. Relevant eligibility criteria were an age range of 18-75 years, a confirmed diagnosis of AS according to the modified New York Criteria, the presence of axial involvement, no peripheral involvement (apart from hips and/or shoulders) and the need for daily treatment with NSAIDs. Eligible subjects entered a wash-out phase of 214 days, during which all NSAIDs and other analgesics were withdrawn. Subjects were suitable for randomisation if they presented with an acute episode of moderate to severe pain at baseline (ie, $\geqslant 40 \mathrm{~mm}$ on a pain visual analogue scale, VAS) and with an increase in pain VAS of $\geqslant 30 \%$ compared to the screening visit after cessation of NSAID treatment.

Present or previous episodes of inflammatory bowel disease or a history of upper gastrointestinal ulcers within the previous year and confirmed by endoscopy were regarded as exclusion criteria.

\section{Study treatment}

Three treatment arms with fixed doses of celecoxib $200 \mathrm{mg}$ once a day, celecoxib $200 \mathrm{mg}$ twice a day, or diclofenac $75 \mathrm{mg}$ slow release (SR) twice a day were used. At baseline, eligible subjects were randomly assigned (ratio 1:1:1) to double-dummy study medication (capsules of celecoxib, diclofenac, and matching placebo) for oral administration over a treatment period of 12 weeks. Concomitant treatments with DMARDs (if used at a stable dose for at least 3 months prior to study start and no change planned during the study period) and prednisolone equivalents of $\leqslant 10 \mathrm{mg} /$ day at stable doses were permitted. In addition, the concomitant administration of proton pump inhibitors (PPI) was allowed at the discretion of the investigators.

\section{Assessment criteria}

Study assessments were performed at screening, baseline, and subsequent treatment visits (weeks 1, 2, 6, and 12). Global pain intensity was measured on a $100-\mathrm{mm}$ pain VAS at all visits. The Ankylosing Spondylitis Assessment Study group 20\% (ASAS 20) response was determined from week 1 onwards. Assessments of core symptoms, functions, and mobility were performed using the Bath Ankylosing Spondylitis Disease Activity Index (BASDAI), Functional Index (BASFI), and Metrology Index (BASMI). ${ }^{14-16}$ In addition, spinal pain (numeric scale with a total sum range of 0-16), nocturnal pain (100-mm VAS), and global assessment of disease activity (numeric scale ranging from
$0=$ "inactive" to $10=$ "highly active") were documented Morning stiffness was assessed in a post-hoc analysis as the mean of BASDAI items 5 and 6. Adverse events (AEs) were documented throughout the study. C-reactive protein was measured and recorded as a biological marker of inflammation.

\section{Statistical analysis}

The aim was to demonstrate the non-inferiority of celecoxib treatment regimens compared to diclofenac. The primary efficacy criterion was the treatment contrast "celecoxib vs diclofenac" for the absolute differences on the global pain intensity VAS at week 12 compared to baseline with a noninferiority boundary of $\delta=10 \mathrm{~mm}$. The sample size estimation, which was performed prior to the study, resulted in a required number of 124 valid subjects per treatment group. When additionally considering protocol deviations and drop-outs, it was decided that a total of 150 subjects per treatment group were to be enrolled.

Starting with the comparison of celecoxib $400 \mathrm{mg}$ and diclofenac, the primary analysis was performed hierarchically in the per protocol (PP) population. Primary and secondary efficacy variables were analysed using several analysis of covariance (ANCOVA) models. For the primary analysis baseline, VAS and age were used as covariates; and sex, treatment and pooled centre as factors. Treatment contrasts were mainly computed with two-sided $95 \%$ CIs for the treatment difference between celecoxib and diclofenac. ASAS 20 responses were analysed using the $\chi^{2}$ test and logistic regression. The safety analysis was performed descriptively; the rates of GI events were compared between the treatment groups using the $\chi^{2}$ test.

\section{RESULTS}

A total of 458 subjects ( $69 \%$ male, $n=317$ ) with a mean age of 44.8 years (range 18-77 years) were randomised and treated (fig 1). The requirements of the sample size estimation (considering a statistical power of $80 \%$ ) were met.

The number of subjects excluded from the PP analysis was comparable between the three treatment groups. Major protocol violations occurring in $\leqslant 5.0 \%$ of subjects in any treatment group were classed as "drop-out for any reason" (apart from drop-out due to lack of efficacy or discontinuation after week 6) and "inappropriately low pain intensity at baseline". Overall, the treatment groups were well balanced with regard to their demographic and baseline characteristics.

The confirmatory analysis (PP population, 373 patients) showed that there was a clinically relevant decrease in the mean pain VAS score over the treatment period of 12 weeks in all treatment groups by (least square (LS) means) $-29.1 \mathrm{~mm}$ on celecoxib $200 \mathrm{mg}$ once a day, (95\% CI: -33.6 to -24.6), $-31.7 \mathrm{~mm}$ on celecoxib $200 \mathrm{mg}$ twice a day (95\% CI: -36.2 to -27.2$)$, and $-32.7 \mathrm{~mm}$ on diclofenac $(95 \% \mathrm{CI}$ : -37.1 to -28.2). LS means for the treatment contrasts were $1.0 \mathrm{~mm}$ for celecoxib $200 \mathrm{mg}$ twice a day vs diclofenac and $3.6 \mathrm{~mm}$ for celecoxib $200 \mathrm{mg}$ once a day vs diclofenac, the corresponding 95\% CIs (two-sided test) were -5.0 to 6.9 and -2.3 to 9.4 , respectively. Thus, non-inferiority of both celecoxib regimens in comparison to diclofenac in terms of pain relief can be concluded, since the upper CI boundaries for the treatment contrasts did not exceed the critical non-inferiority threshold of $10 \mathrm{~mm}$. Sensitivity analyses in the full analysis set (FAS, similar to an intent-to-treat population; table 1) and alternative ANCOVA models consistently supported the results obtained in the primary analysis. 


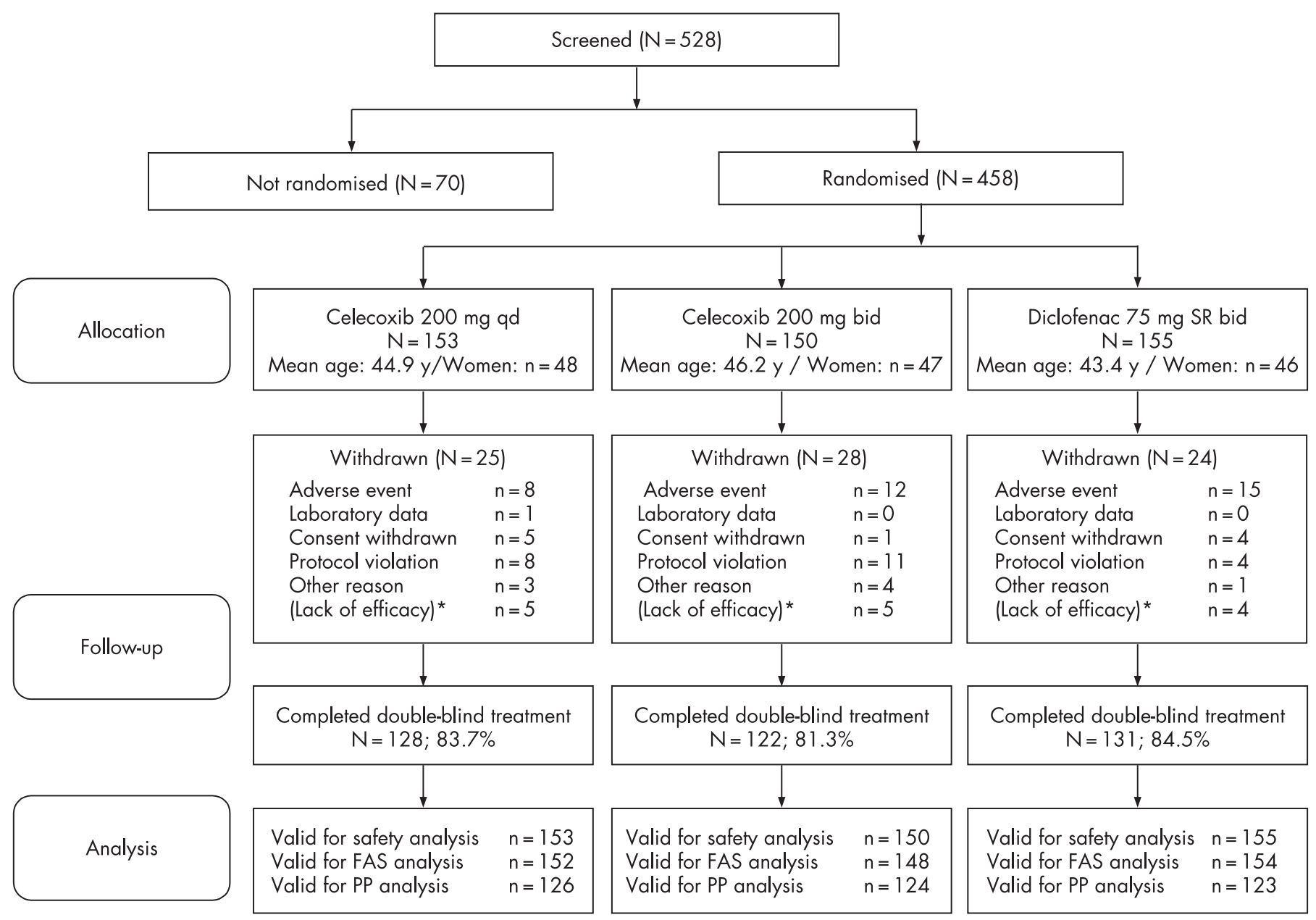

Figure 1 Disposition of study subjects. * Note that the analysis of premature withdrawal was based on the allocation to one primary reason for withdrawal in the case of multiple reasons. Fourteen patients had an additional specification of "lack of efficacy" but were allocated to another (primary) category. Essentially, the number of patients with "lack of efficacy" was comparable between the treatment groups. $y=y e a r s$.

Generally, the explorative analyses of the other secondary efficacy variables (table 1) indicated that there were no statistically significant differences (ie, $95 \%$ CIs for treatment differences not including the null value) between celecoxib $200 \mathrm{mg}$ twice a day and diclofenac in any of the performed analyses of the secondary efficacy variables from week 1 onwards, whereas some statistically significant differences between celecoxib $200 \mathrm{mg}$ once a day and diclofenac were observed at week 12 (ASAS 20 response, BASDAI, spinal pain, and nocturnal pain). The logistic regression analysis of the ASAS 20 response at week 12 indicated a statistical difference between diclofenac and celecoxib $200 \mathrm{mg}$ once a day in favour of diclofenac (PP population). Likewise, the BASDAI total score had improved to a similar extent in the celecoxib $200 \mathrm{mg}$ twice a day and diclofenac groups, whereas the improvement in the celecoxib $200 \mathrm{mg}$ once a day group was numerically weaker (and the difference to diclofenac was statistically significant). A comparable result was seen for the morning stiffness with improvements (LS mean) by -1.84 points on diclofenac, -1.76 points on celecoxib $200 \mathrm{mg}$ twice a day and -1.07 points on celecoxib $200 \mathrm{mg}$ once a day $(95 \% \mathrm{CI}$ for treatment contrast "celecoxib $200 \mathrm{mg}$ vs diclofenac": 0.31 to 1.23 ).

There was a rapid reduction of pain in all three groups after just 1 week of treatment, with further improvement through to week 12. For both the celecoxib $200 \mathrm{mg}$ twice a day and the diclofenac groups, an ASAS 20 response was achieved by $40 \%$ after 1 week but with a further steady increase to about $50 \%$ at week 2 and to $60 \%$ at week 6 , reflecting that this composite outcome measure does not only measure pain, but also function, inflammation, and patient's global disease activity.

The reductions in the BASDAI and BASFI mean scores over time are displayed in fig $2 \mathrm{~A}, \mathrm{~B}$. There was no change in spinal mobility as measured by the BASMI (table 1). Interestingly, there was a significant within-group decrease in the CRP value over the 12 weeks in all three treatment groups (table 1).

A total of 251 subjects (54.8\%) experienced treatmentemergent AEs (TEAEs, table 2). No deaths were reported. The rate of serious TEAEs (seven subjects involved) was comparable between the three treatment groups. The serious AEs were angina pectoris, dyspnoea, sudden hearing loss in the celecoxib $200 \mathrm{mg}$ once a day, deterioration of AS, familial Mediterranean fever in the celecoxib $200 \mathrm{mg}$ twice a day, and myocardial infarction and tinnitus in the diclofenac treatment group, but only one of these serious TEAEs was considered drug-related by the investigator (dyspnoea on celecoxib $200 \mathrm{mg}$ once a day).

Generally, the incidences of gastrointestinal TEAEs (table 2) were numerically higher with diclofenac $(28.4 \%)$ than with celecoxib at either dose (200 mg once a day: $15.0 \%, 200 \mathrm{mg}$ twice a day: $16.7 \%$ ) and the statistical comparison indicated a significant difference between the three treatment groups 
Table 1 Changes from baseline in the efficacy variables after 12 weeks of treatment*

\begin{tabular}{|c|c|c|c|}
\hline \multirow[b]{2}{*}{ Variable } & \multicolumn{2}{|l|}{ Celecoxib } & \multirow{2}{*}{$\begin{array}{l}\text { Diclofenac } \\
75 \mathrm{mg} \text { twice a day }\end{array}$} \\
\hline & $200 \mathrm{mg}$ once a day & 200 mg twice a day & \\
\hline \multicolumn{4}{|l|}{ VAS pain $(0-100 \mathrm{~mm})$} \\
\hline Baseline & $65.6(14.9)$ & $68.1(16.4)$ & $64.3(16.6)$ \\
\hline Week 12 & $37.4(25.6)$ & $38.7(24.9)$ & $33.8(27.1)$ \\
\hline Mean change from baseline $\dagger$ & $-28.2(27.2)$ & $-29.8(25.1)$ & $-30.8(25.6)$ \\
\hline LS mean treatment contrast & $2.9(2.7)$ & $2.1(2.8)$ & NA \\
\hline $95 \% \mathrm{Cl}$ for the treatment contrast & -2.4 to 8.2 & -3.3 to 7.6 & NA \\
\hline \multicolumn{4}{|l|}{ ASAS 20 response (PP population) } \\
\hline Week $12\left(\chi^{2}\right.$ test: 0.039$)$ & $\mathrm{n}=58(46.0 \%)$ & $\mathrm{n}=74(59.7 \%)$ & $\mathrm{n}=74(60.2 \%)$ \\
\hline Parameter estimator for treatment & -0.448 & 0.267 & NA \\
\hline $95 \% \mathrm{Cl}$ for parameter estimator & -0.758 to -0.137 & -0.050 to 0.584 & NA \\
\hline Odds ratio (celecoxib/diclofenac) & 0.533 & 1.090 & NA \\
\hline \multicolumn{4}{|l|}{ BASDAI (0-10) } \\
\hline Baseline & $4.66(1.63)$ & $4.86(1.64)$ & $4.75(1.78)$ \\
\hline Week 12 & $3.69(2.19)$ & $3.55(2.07)$ & $3.27(2.21)$ \\
\hline Mean change from baseline $\dagger$ & $-0.99(2.11)$ & $-1.32(1.72)$ & $-1.48(1.76)$ \\
\hline LS mean treatment contrast: & $0.42(0.20)$ & $0.11(0.20)$ & NA \\
\hline $95 \% \mathrm{Cl}$ for the treatment contrast & 0.03 to 0.81 & -0.29 to 0.51 & NA \\
\hline \multicolumn{4}{|l|}{ BASFI $(0-10)$} \\
\hline Baseline & $4.5(2.3)$ & $4.5(2.2)$ & $4.2(2.3)$ \\
\hline Week 12 & $3.7(2.6)$ & $3.6(2.5)$ & $3.4(2.5)$ \\
\hline Mean change from baseline $\dagger$ & $-0.8(2.0)$ & $-0.9(1.5)$ & $-0.9(1.8)$ \\
\hline LS mean treatment contrast & $0.1(0.2)$ & $-0.0(0.2)$ & NA \\
\hline $95 \% \mathrm{Cl}$ for the treatment contrast & -0.3 to 0.5 & -0.4 to 0.3 & NA \\
\hline \multicolumn{4}{|l|}{ GA disease activity, subjects $(0-10)$} \\
\hline Baseline & $6.1(1.8)$ & $6.5(1.7)$ & $6.1(1.8)$ \\
\hline Week 12 & $4.1(2.4)$ & $4.3(2.5)$ & $3.8(2.6)$ \\
\hline Mean change $\dagger$ & $-2.0(2.7)$ & $-2.2(2.5)$ & $-2.3(2.6)$ \\
\hline LS mean treatment contrast: & $0.3(0.3)$ & $0.3(0.3)$ & NA \\
\hline $95 \% \mathrm{Cl}$ for the treatment contrast & -0.2 to 0.8 & -0.2 to 0.8 & NA \\
\hline \multicolumn{4}{|l|}{ BASMI $(0-10)$} \\
\hline Baseline & $4.1(2.5)$ & $3.8(2.3)$ & $3.8(2.2)$ \\
\hline Week 12 & $3.8(2.6)$ & $3.5(2.4)$ & $3.4(2.2)$ \\
\hline Mean change $\dagger$ & $-0.3(1.4)$ & $-0.3(1.4)$ & $-0.5(1.3)$ \\
\hline LS mean treatment contrast & $0.1(0.1)$ & $0.1(0.1)$ & NA \\
\hline $95 \% \mathrm{Cl}$ for the treatment contrast & -0.1 to 0.4 & -0.1 to 0.4 & NA \\
\hline \multicolumn{4}{|l|}{ C-reactive protein $(\mathrm{mg} / \mathrm{L})$} \\
\hline Baseline & $12.6(14.3)$ & $13.4(13.8)$ & $13.8(14.6)$ \\
\hline Week 12 & $10.6(10.7)$ & $9.7(12.7)$ & $10.7(9.8)$ \\
\hline Mean change $\dagger$ & $-2.2(11.3)$ & $-3.4(11.3)$ & $-3.5(10.0)$ \\
\hline LS mean treatment contrast & $0.6(1.0)$ & $-0.1(1.0)$ & NA \\
\hline $95 \% \mathrm{Cl}$ for the treatment contrast & -1.4 to 2.5 & -2.1 to 1.9 & NA \\
\hline
\end{tabular}

*Data of the FAS with "last observation carried forward" approach are given apart from the ASAS 20 response (here: PP population). For continuous variables: baseline, week 12 values and related changes are the mean (SD); values of treatment contrasts are LS means (SEM). For ASAS 20 response: Logistic progression is described with parameter estimator for treatment compared to diclofenac after adjusting for baseline VAS, sex, centre, and age; results represent the comparison with the reference class "diclofenac".

$\dagger$ Calculated as post-baseline values minus baseline values.

\#Calculated as the difference in the respective celecoxib group minus the difference in the diclofenac group; derived from pooled centre ANCOVA with baseline VAS and age as covariates and treatment, sex and centre as factors.

ASAS 20, Ankylosing Spondylitis Assessment Study group 20\%; BASDAl, Bath Ankylosing Spondylitis Disease Activity Index

BASFI, Bath Ankylosing Spondylitis Functional Index; BASMI, Bath Ankylosing Spondylitis Metrology Index; FAS, full analysis set;

$\mathrm{GA}$, global assessment; LS, least square; NA, not applicable; $\mathrm{PP}$, per protocol; VAS, visual analogue scale.

$\left(p=0.006, \chi^{2}\right.$ test $)$. Furthermore, the descriptive $p$ values for the direct between-group comparisons versus diclofenac were statistically significant both in favour of celecoxib $200 \mathrm{mg}$ once a day $(p=0.005)$ and $200 \mathrm{mg}$ twice a day $(p=0.014)$. The differentiation between upper and lower GI events showed similar results with statistically significant differences in the post-hoc tests that were always in favour of celecoxib $200 \mathrm{mg}$ (once or twice a day) compared with diclofenac (table 2). A comparable percentage of patients were treated with a PPI in all three groups: $17.6 \%, 12.0 \%$, and $14.8 \%$ of patients in the celecoxib $200 \mathrm{mg}$ once a day, celecoxib $200 \mathrm{mg}$ twice a day, and diclofenac SR $75 \mathrm{mg}$ twice a day group, respectively.

TEAEs pertaining to the System Organ Class (SOC) "cardiac disorders" were observed in six $(1.3 \%)$ subjects (three subjects each on treatment with celecoxib $200 \mathrm{mg}$ once a day (angina pectoris, cardiovascular disorder, tachycardia) and diclofenac (angina pectors, myocardial infarction, tachycardia)). Drug-related cardiac AEs occurred in three subjects (celecoxib $200 \mathrm{mg}$ : tachycardia; diclofenac: angina pectoris and tachycardia). Hypertension was observed in three subjects (celecoxib $200 \mathrm{mg}$ 


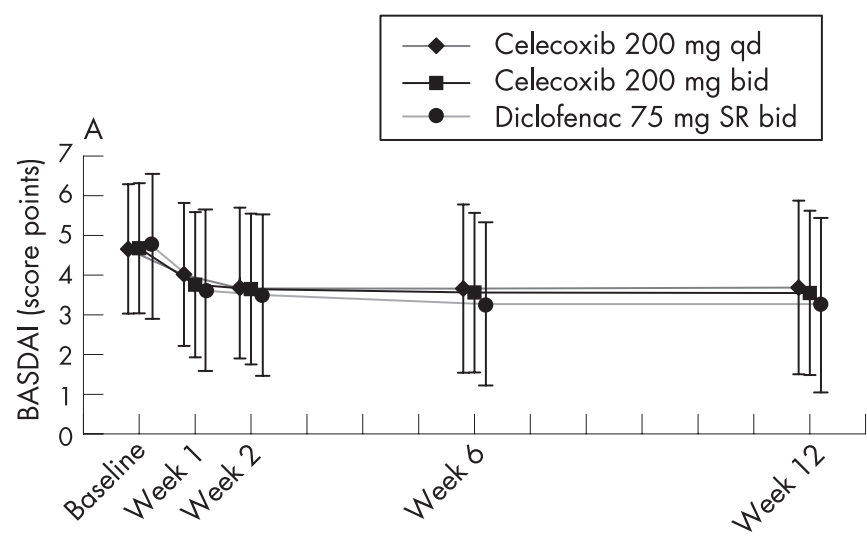

Assessment time

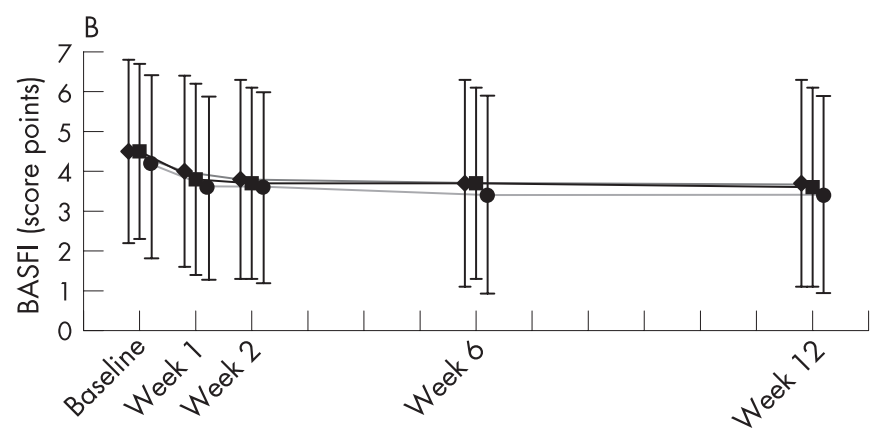

Assessment time

Figure 2 A. Course of Bath Ankylosing Spondylitis Disease Activity Index (BASDAl) over 12 weeks (wk) of treatment; full analysis set (FAS). The $95 \%$ Cls for the least square (LS) mean changes within the three treatment groups (from baseline to week 12) did not include the 0 value, thereby indicating a statistically significant reduction in each of the treatment groups (see also table 1). B. Course of Bath Ankylosing Spondylitis Functional Index (BASFI) over 12 weeks of treatment; FAS. The $95 \%$ Cls for the LS mean changes within the three treatment groups (from baseline to week 12) did not include the 0 value, thereby indicating a statistically significant reduction in each of the treatment groups (see also table 1).

once a day in one patient, diclofenac in two patients). Diclofenac had worse hepatic tolerability than celecoxib in terms of AEs of transaminase increase. The occurrence of headache as a TEAE was unexpectedly high in all of the treatment groups.

The proportion of subjects with premature withdrawal from the study due to any AEs was numerically, but nonsignificantly, higher in the diclofenac group $(n=15,9.7 \%)$ than in the celecoxib $200 \mathrm{mg}$ once a day and twice a day groups ( $\mathrm{n}=8,5.2 \%$ and $\mathrm{n}=12,8.0 \%$, respectively). The most common AEs that led to premature withdrawal were classified within the SOCs "musculoskeletal and connective tissue disorders" and "gastrointestinal disorders" (10 subjects each in total). The gastrointestinal TEAEs leading to premature withdrawal occurred in three subjects $(2.0 \%)$ on celecoxib $200 \mathrm{mg}$ twice a day (events: duodenal ulcer, haemorrhoidal haemorrhage, oral mucosal blistering, and swollen tongue) and in seven subjects $(4.5 \%)$ on diclofenac (events: upper abdominal pain, diarrhoea NOS, diarrhoea haemorrhagic, epigastric discomfort, haemorrhoidal haemorrhage, and nausea).

\section{DISCUSSION}

The results of our study demonstrated that clinically relevant improvement was achieved at week 12 in all three treatment groups, as indicated by the reduction in the primary efficacy variable pain VAS. In the primary efficacy analysis, celecoxib at either dose proved to be comparable in efficacy to diclofenac for pain alleviation in subjects with AS after 12 weeks of treatment, thereby confirming the study hypothesis of non-inferiority of celecoxib compared with diclofenac in terms of pain reduction and underlining the therapeutic value of celecoxib within the options available for the management of AS.

The results concerning the primary variable "pain VAS" in subjects on celecoxib were consistent with other studies. ${ }^{12} 13$ The observed effect of celecoxib $200 \mathrm{mg}$ once a day on the pain VAS in this study at week 6 (FAS: mean (SD) change from baseline by $-27.4(24.3) \mathrm{mm}$ ) was consistent with the effects seen in the celecoxib $100 \mathrm{mg}$ twice a day arm (mean change by 27 (30) $\mathrm{mm}$ from baseline $70(16) \mathrm{mm}$ ) in a 6-week study of celecoxib vs ketoprofen. ${ }^{12}$ Likewise, a 12 -week randomised controlled trial comparing celecoxib to naproxen yielded results for the reduction in the pain VAS over 12 weeks that were comparable to our results; the efficacy celecoxib at either dose was comparable to that of naproxen. ${ }^{13}$

The primary efficacy analysis based on the pain VAS indicated that both at doses of $200 \mathrm{mg}$ twice a day and $200 \mathrm{mg}$ once a day celecoxib was non-inferior compared to diclofenac. In the explorative analyses of the secondary efficacy endpoints, celecoxib $200 \mathrm{mg}$ twice a day was always non-inferior to diclofenac, whereas celecoxib $200 \mathrm{mg}$ once a day did not show non-inferiority to diclofenac in some of the secondary efficacy analyses (eg, with parameters reflecting inflammation (such as morning stiffness) or with the composite outcome measurement ASAS 20 response, which includes-apart from the domain "pain" - other domains such as inflammation as well). In addition, the descriptive comparison between the two celecoxib arms consistently showed that the mean treatment effects were numerically stronger on celecoxib $200 \mathrm{mg}$ twice a day than on celecoxib $200 \mathrm{mg}$ once a day in most of the analysed efficacy variables (this also applies to the parameters of inflammation), thereby suggesting a certain dosedependent effect and a potentially better treatment outcome with the higher dose of celecoxib.

A recent study compared another COX-2 selective NSAID, etoricoxib, with naproxen or placebo in the treatment of active AS subjects. ${ }^{17}$ Both drugs were clearly superior to placebo, but etoricoxib (both at a doses of 90 and of $120 \mathrm{mg} /$ day) was more effective than naproxen $(1000 \mathrm{mg} /$ day $)$ in some of the efficacy variables. The ASAS 20 response rates of $64.7 \%$ and $64.8 \%$ in the two etoricoxib arms were similar to the response rates in the $400 \mathrm{mg}$ celecoxib and $150 \mathrm{mg}$ diclofenac groups in our study. In another post-hoc subgroup analysis, the ASAS 20 response was even, although non-significantly, higher in patients without concomitant peripheral arthritis; a subgroup that is more similar to the patients studied here. ${ }^{18}$

Importantly, both celecoxib dose regimens were safe and well tolerated with better GI tolerability than diclofenac. Similar observations have already been reported in previous diclofenaccontrolled studies. ${ }^{19} 20$ Furthermore, there were no signs of a dose-dependent increase in the overall and GI toxicity of celecoxib, which was consistent with the findings of a previous meta-analysis. ${ }^{5}$

The results of the present study did not indicate any cardiovascular-related safety concerns with celecoxib administered over a period of 12 weeks. However, it should be noted that the number of subjects was too small and the duration of treatment too short to allow further conclusions.

There is an ongoing debate whether NSAIDs should only be used periodically on demand or rather continuously. The 
Table 2 Overall treatment-emergent adverse event (TEAE) experience (safety population)

\begin{tabular}{|c|c|c|c|}
\hline & \multicolumn{2}{|l|}{ Celecoxib } & \multirow{2}{*}{$\begin{array}{l}\text { Diclofenac SR } \\
75 \mathrm{mg} \text { twice a day } \\
\text { ( } \mathrm{n}=155)\end{array}$} \\
\hline & $\begin{array}{l}200 \mathrm{mg} \text { once a day } \\
(\mathrm{n}=153)\end{array}$ & $\begin{array}{l}200 \mathrm{mg} \text { twice a day } \\
(\mathrm{n}=150)\end{array}$ & \\
\hline Subjects with any TEAEs: & $92(60.1 \%)$ & $68(45.3 \%)$ & $91(58.7 \%)$ \\
\hline No. of events & 180 & 153 & 218 \\
\hline \multicolumn{4}{|l|}{ Intensity:* } \\
\hline Mild & $34(22.2 \%)$ & $24(16.0 \%)$ & $36(23.2 \%)$ \\
\hline Moderate & $43(28.1 \%)$ & $35(23.3 \%)$ & $43(27.7 \%)$ \\
\hline Severe & $15(9.8 \%)$ & $9(6.0 \%)$ & $12(7.7 \%)$ \\
\hline Subjects with drug-related TEAEs & $29(19.0 \%)$ & $31(20.7 \%)$ & $41(26.5 \%)$ \\
\hline Subjects with serious TEAEs & $3(2.0 \%)$ & $2(1.3 \%)$ & $2(1.3 \%)$ \\
\hline Subjects with drug-related serious TEAEs & $1(0.7 \%)$ & 0 & 0 \\
\hline $\begin{array}{l}\text { Subjects with premature withdrawal of study } \\
\text { medication due to TEAEs }\end{array}$ & $8(5.2 \%)$ & $12(8.0 \%)$ & $15(9.7 \%)$ \\
\hline Subjects with gastrointestinal TEAEs: $\dagger$ & $23(15.0 \%)$ & $25(16.7 \%)$ & $44(28.4 \%)$ \\
\hline Upper GI TEAEs§ & $10(6.5 \%)$ & $11(7.3 \%)$ & $28(18.1 \%)$ \\
\hline Lower GI TEAEs§ & $9(5.9 \%)$ & $5(3.3 \%)$ & $20(12.9 \%)$ \\
\hline \multicolumn{4}{|l|}{ Most common AEs: } \\
\hline Abdominal distension & $3(2.0 \%)$ & 0 & $1(0.6 \%)$ \\
\hline Abdominal pain NOS & $1(0.7 \%)$ & $1(0.7 \%)$ & $4(2.6 \%)$ \\
\hline Abdominal pain upper & $5(3.3 \%)$ & $5(3.3 \%)$ & $14(9.0 \%)$ \\
\hline Diarrhoea NOS & $6(3.9 \%)$ & $4(2.7 \%)$ & $15(9.7 \%)$ \\
\hline Epigastric discomfort & 0 & $1(0.7 \%)$ & $6(3.9 \%)$ \\
\hline Gastritis NOS & $1(0.7 \%)$ & $4(2.7 \%)$ & $2(1.3 \%)$ \\
\hline Nausea & 0 & $2(1.3 \%)$ & $5(3.2 \%)$ \\
\hline Stomach discomfort & $4(2.6 \%)$ & $1(0.7 \%)$ & $4(2.6 \%)$ \\
\hline Toothache & $4(2.6 \%)$ & $3(2.0 \%)$ & $3(1.9 \%)$ \\
\hline Fatigue & $3(2.0 \%)$ & $3(2.0 \%)$ & $1(0.6 \%)$ \\
\hline Influenza-like illness & $8(5.2 \%)$ & $4(2.7 \%)$ & $2(1.3 \%)$ \\
\hline ALAT increased & 0 & 0 & $6(3.9 \%)$ \\
\hline Arthralgia & $2(1.3 \%)$ & $3(2.0 \%)$ & 0 \\
\hline AS aggravated & $6(3.9 \%)$ & $5(3.3 \%)$ & $2(1.3 \%)$ \\
\hline Dizziness & $2(1.3 \%)$ & $1(0.7 \%)$ & $5(3.2 \%)$ \\
\hline Headache & $30(19.6 \%)$ & $22(14.7 \%)$ & $34(21.9 \%)$ \\
\hline Cough & $1(0.7 \%)$ & $3(2.0 \%)$ & $2(1.3 \%)$ \\
\hline Nasopharyngitis & $5(3.3 \%)$ & $5(3.3 \%)$ & $4(2.6 \%)$ \\
\hline Pharyngitis & $5(3.3 \%)$ & $1(0.7 \%)$ & 0 \\
\hline
\end{tabular}

*Intensity given by subjects, the most intense event experienced per subject was considered for classification. No deaths were reported in this study.

$\uparrow$ According to MedDRA System Organ Class "gastrointestinal disorders". Descriptive $p$ values ( $\chi^{2}$ test) for between-group differences were $p=0.005$ for celecoxib $200 \mathrm{mg}$ once a day vs diclofenac and $p=0.014$ for celecoxib 200 mg twice a day vs diclofenac.

§Included were the terms (abdominal pain upper; epigastric discomfort; gastritis NOS; nausea; stomach discomfort) as "upper" GI events and (abdominal distension, abdominal pain NOS, diarrhoea) as "lower" Gl events. The four between-group comparisons vs diclofenac were always in favour of celecoxib at either dose (each $\mathrm{p}<0.05 ; \chi^{2}$ test).

flncidence of $\geqslant 2 \%$ at MedDRA preferred term level in any treatment group, sorted alphabetically.

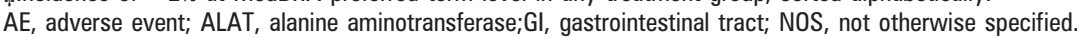

reasons for this debate are the problematic GI tolerability of conventional NSAIDs and the question, whether NSAIDs may have any long-term disease modifying effect. ${ }^{21}$ However, a recent study showed that, in comparison to NSAIDs on demand, continuously applied NSAIDs over 2 years may have the capacity to stop radiological progression in the spine of AS subjects, thereby underlining the need for safe NSAIDs that are suitable for continuous long-term treatment. ${ }^{22}$

The results of this study demonstrated similar efficacy, but with a more favourable GI safety profile for celecoxib than diclofenac and, therefore, suggest that celecoxib should be added to the therapeutic armamentarium for the management of AS. Our results underline the efficacy of NSAIDs in the treatment of AS and suggest that a higher dosage might be necessary in some subjects to achieve an optimum therapeutic result. Thus, a suitable strategy for treating AS patients would be to start celecoxib treatment with $200 \mathrm{mg}$ once a day initially with the option to increase the dose to $200 \mathrm{mg}$ twice a day in the case of insufficient pain relief or persisting inflammatory activity.

Acknowledgements: Study investigators, in addition to the authors JS, TK, MR and AK were as follows: S. Alsalameh, R. Alten, M. Aurich, K. Babinsky, W. Biewer, G-R. Burmester, R. Dockhorn, E. Edelmann, G. Gauler, A. Gause, A. Gräßler, E. GromnicaIhle, J. Häntsch, R. Haux, B. Heilig, W. Heitz, P. Hrdlicka, W. Hungerberg, J. Kögler, G. Kramer, R. Kurthen, C. Lohmüller, K-P. Martin, L. Meier, A. Melzer, U. Müller-Ladner, R. Nischik, D. Richter, E. Röther, U. Schendel, S. Schewe, H-J. Schmidt, U. Schoo, A. Schrepler-Konya, H-E. Schröder, C. Stille, H. Strothmeyer, A. Teipel, U. von Hinüber, G. Voss, A. Wagenitz and J. Wendler. The authors are also grateful to Wilfried Collet, Thomas Fischer, Bernd Graulich and Claudia Stumpf from Winicker Norimed Nürnberg, Germany for their advice and continuous support of the study.

Funding: This study was sponsored by Pfizer Pharma GmbH, Germany.

Competing interests: SS, ER and MM were employed by Pfizer, and the study was supported by Pfizer. No further conflict of interest. 


\section{REFERENCES}

1. Amor B, Dougados M, Listrat V, Menkes C, Roux H, Benhamou C, et al. Are classification criteria for spondylarthropathy useful as diagnostic criteria? Rev Rhum Engl Ed 1995;62:10-15.

2. Zochling J, van der Heijde D, Burgos-Vargas R, Collantes E, Davis JC Jr, Dijkmans B, et al. ASAS/EULAR recommendations for the management of ankylosing spondylitis. Ann Rheum Dis 2006;65:442-52.

3. Braun J, Davis J, Dougados M, Sieper J, van der Linden S, van der Heijde D. First update of the international ASAS consensus statement for the use of anti-TNF agents in patients with ankylosing spondylitis. Ann Rheum Dis 2006;65:316-20.

4. Silverstein F, Faich G, Goldstein J, Simon L, Pincus T, Whelton A, et al. Gastrointestinal toxicity with celecoxib vs nonsteroidal anti-inflammatory drugs for osteoarthritis and rheumatoid arthritis: the CLASS study: A randomized controlled trial. Celecoxib Long-term Arthritis Safety Study. JAMA 2000;284:1247-55.

5. Bensen W, Zhao S, Burke T, Zabinski R, Makuch R, Maurath C, et al. Upper gastrointestinal tolerability of celecoxib, a COX-2 specific inhibitor, compared to naproxen and placebo. J Rheumatol 2000;27:1876-83.

6. Goldstein J, Silverstein F, Agrawal N, Hubbard R, Kaiser J, Maurath C, et al. Reduced risk of upper gastrointestinal ulcer complications with celecoxib, a novel COX-2 inhibitor. Am J Gastroenterol 2000;95:1681-90.

7. Brophy JM. Celecoxib and cardiovascular risks. Expert Opin Drug Saf 2005;4:1005-15.

8. Singh G, Fort JG, Goldstein JL, Levy RA, Hanrahan PS, Bello AE, et al. Celecoxib versus naproxen and diclofenac in osteoarthritis patients: SUCCESS-I Study. Am J Med 2006;119:255-66.

9. Arber N, Eagle C, Spicak J, Racz I, Dite P. Hajer J, et al. Celecoxib for the prevention of colorectal adenomatous polyps. N Engl J Med 2006;355:885-95.

10. Bertagnolli MM, Eagle CJ, Zauber AG, Redston M, Solomon SD, Kim K, et al. Celecoxib for the prevention of sporadic colorectal adenomas. N Engl J Med 2006;355:873-84.

11. McGettigan P, Henry D. Cardiovascular risk and inhibition of cyclooxygenase: a systematic review of the observational studies of selective and nonselective inhibitors of cyclooxygenase 2. JAMA 2006;296:1633-44.

12. Dougados M, Behier J, Jolchine I, Calin A, van der Heijde D, Olivieri I, et al. Efficacy of celecoxib, a cyclooxygenase 2-specific inhibitor, in the treatment of ankylosing spondylitis: a six-week controlled study with comparison against placebo and against a conventional nonsteroidal antiinflammatory drug. Arthritis Rheum 2001;44:180-5.

13. Barkhuizen A, Steinfeld S, Robbins J, West C, Coombs J, Zwillich S. Celecoxib is efficacious in treating the signs and symptoms of ankylosing spondylitis. Ann Rheum Dis 2005;64(Suppl 3):338

14. Garrett S, Jenkinson T, Kennedy L, Whitelock H, Gaisford P, Calin A. A new approach to defining disease status in ankylosing spondylitis: the Bath Ankylosing Spondylitis Disease Activity Index. J Rheumatol 1994;21:2286-91.

15. Calin A, Garrett S, Whitelock H, Kennedy L, O'Hea J, Mallorie P, et al. A new approach to defining functional ability in ankylosing spondylitis: the development of the Bath Ankylosing Spondylitis Functional Index. J Rheumatol 1994;21:2281-5.

16. Jenkinson T, Mallorie P, Whitelock H, Kennedy L, Garrett S, Calin A. Defining spinal mobility in ankylosing spondylitis (AS). The Bath AS Metrology Index. J Rheumatol 1994;21:1694-8.

17. van der Heijde D, Baraf $H$, Ramos-Remus $C$, Calin A, Weaver A, Schiff M, et al. Evaluation of the efficacy of etoricoxib in ankylosing spondylitis: results of a fifty-two week, randomized, controlled study. Arthritis Rheum 2005:52:1205-15.

18. Gossec L, van der Heijde D, Melian A, Krupa DA, James MK, Cavanaugh PF, et al. Efficacy of cyclo-oxygenase-2 inhibition by etoricoxib and naproxen on the axial manifestations of ankylosing spondylitis in the presence of peripheral arthritis. Ann Rheum Dis 2005;64:1563-7.

19. Emery P, Zeidler H, Kvien T, Guslandi M, Naudin R, Stead H, et al. Celecoxib versus diclofenac in long-term management of rheumatoid arthritis: randomised double-blind comparison. Lancet 1999;354:2106-11.

20. McKenna F, Borenstein D, Wendt H, Wallemark C, Lefkowith J, Geis G. Celecoxib versus diclofenac in the management of osteoarthritis of the knee. Scand J Rheumatol 2001;30:11-18.

21. Dougados M, Dijkmans B, Khan M, Maksymowych W, van der Linden S, Brandt J. Conventional treatments for ankylosing spondylitis. Ann Rheum Dis 2002;61/Suppl 3):iii40-50.

22. Wanders A, Heijde D, Landewe R, Behier J, Calin A, Olivieri I, et al. Nonsteroidal antiinflammatory drugs reduce radiographic progression in patients with ankylosing spondylitis: a randomized clinical trial. Arthritis Rheum 2005;52:1756-65.

\section{Access the latest content chosen by our Editors}

BMJ Journals editors select an article from each issue to be made free online immediately on publication. Other material is free after 12 months to non-subscribers. Access the Editor's Choice from the home page - or expand your horizons and see what the other BMJ Journals editors have chosen by following the links on any BMJ Journal home page. 